\title{
Modal structure design in refractive capillary optical fibers
}

\author{
Ryszard S. Romaniuk* \\ Institute of Electronic Systems, Warsaw University of Technology, Nowowiejska 15/19, 00-665 Warszawa,
}

Received March 14, 2010; accepted March 20, 2010; published March 31, 2010

\begin{abstract}
The work presents the design of a modal structure in refractive, non-photonic, singlemode and low-mode capillary optical fibers (COF). A classical dispersion eigenequation was used to calculate the characteristics in a depressed cladding COF. The results were used for the design of COFs with preset modal characteristics for sensors, chemical microfluidics and photonic functional components.
\end{abstract}

Designing an instrumentation oriented optical fibre concerns geometry, refraction, modal structure and sensitivity to external reactions. The design process of a COF may embrace the following issues [1-6]: maintaining chemical capillary properties while adding photonic functions or vice versa adding a chemical function to the defined COF, fitting a COF to discriminative measurement of physical or thermal values, filling a COF with nonlinear media, maximization of optical power in the singlemode dark hollow beam (DHB), conversion of DHB to Gaussian and back, compensation or correction of dispersion and/or nonlinearity, plasmonic resonant optics at the modified inner surface of a COF, effective optical pumping and active COF, co-transmission of light and material deBroglie wave, microfluidic MOEMS with COF. Each of these fields require a different COF such as: high or low refraction, step or gradient, micro or nanocapillary, single or multiple capillary, conditioned or unconditioned inner surface. Geometry is determined during the hot stage of the pulling process and depends on the preform characteristics. Refraction is determined by constituent glasses and diffusion. Modal structure is determined by the two above factors and the source. The design of a modal structure in a COF is not a trivial issue. The reason is the big dynamics of the manufacturing process, lasting only a few ms, thus a difficulty of the expected translation of the design parameters to the final product.

The dispersion eigenequation of a depressed cladding $\mathrm{COF}$ is $\mathrm{W}=0$, where $\mathrm{W}$ equals

$\left|\begin{array}{cccccc}I_{m}\left(v r_{c}\right) & -J_{m}\left(u r_{c}\right) & -Y_{m}\left(u r_{c}\right) & 0 & 0 & 0 \\ v I_{m}\left(v r_{c}\right) & -u J_{m}\left(u r_{c}\right) & -u Y_{m}\left(u r_{c}\right) & 0 & 0 & 0 \\ 0 & J_{m}\left(u r_{r}\right) & Y_{m}\left(u r_{r}\right) & -I_{m}\left(w r_{r}\right) & -K_{m}\left(w r_{r}\right) & 0 \\ 0 & -u J_{m}\left(u r_{r}\right) & -u Y_{m}^{\prime}\left(u r_{r}\right) & -w K_{m}\left(w r_{r}\right) & -K_{m}\left(w r_{d}\right) & 0 \\ 0 & 0 & 0 & I_{m}\left(w r_{d}\right) & K_{m}\left(w r_{d}\right) & -K_{m}\left(s r_{d}\right) \\ 0 & 0 & 0 & w I_{m}^{\prime}\left(w r_{d}\right) & w K_{m}^{\prime}\left(w r_{d}\right) & -s K_{m}\left(s r_{d}\right)\end{array}\right|$

where $\mathrm{I}_{\mathrm{m}}{ }^{\prime}(\mathrm{ur})=\partial \mathrm{I} / \partial \mathrm{r}$, and the subscripts: c-capillary, r-core, p-cladding, d-depression. Fig.1-5 present numerical

*E-mail: rrom@ise.pw.edu.pl solutions to this equation, where $\mathrm{B}=\mathrm{sqrt}\left[\beta^{2} / \mathrm{k}^{2}-\mathrm{n}_{\mathrm{p}}{ }^{2} / \mathrm{NA}^{2}\right]$ is a normalized propagation constant.

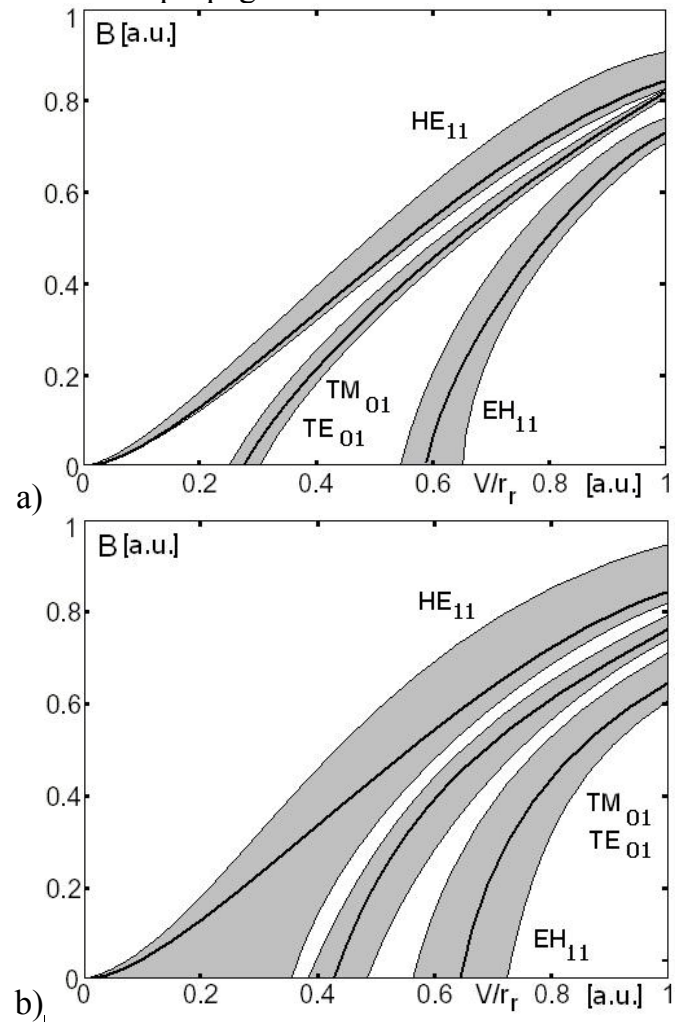

Fig.1. Modal dispersion as a function of normalized frequency for a basic COF (a), and depressed cladding DCOF (b); $\lambda=800 \mathrm{~nm}, \mathrm{~d}_{\mathrm{c}}=3,5 \mu \mathrm{m}, \mathrm{d}_{\mathrm{r}}=2 \mathrm{r}_{\mathrm{r}}=10 \mu \mathrm{m}, \Delta \mathrm{n}_{\mathrm{rp}}=0,2 \%, \mathrm{n}_{\mathrm{p}}=1,50$.

The results were presented for real, practically used sets of fiber manufacturing parameters. The grey area shows the allowed changeability of process controls resulting in different values of $N A, r_{r}, V, \Delta n$. Fig.1 was simplified to avoid too big overlapping of the areas. In reality, the areas do overlap and individual modes are hardly distinguishable, creating groups of similar dispersion. $\mathrm{HE}_{11}$ mode of a basic COF has zero cut-off non-depending on the refraction and geometry. In practice, the mode is not propagated for certain ranges of fiber parameters. The $\mathrm{HE}_{11}$ mode cut-off of a depressed cladding $\mathrm{COF}$ may be nonzero. The $\mathrm{HE}_{11}$ curve nears faster to zero in a fiber with higher NA. The distance between the fundamental mode and the group of next modes is bigger in a COF of smaller NA. A DCOF 
enables more control of fundamental mode separation. This is illustrated in Fig. 1 by the width of the grey areas.

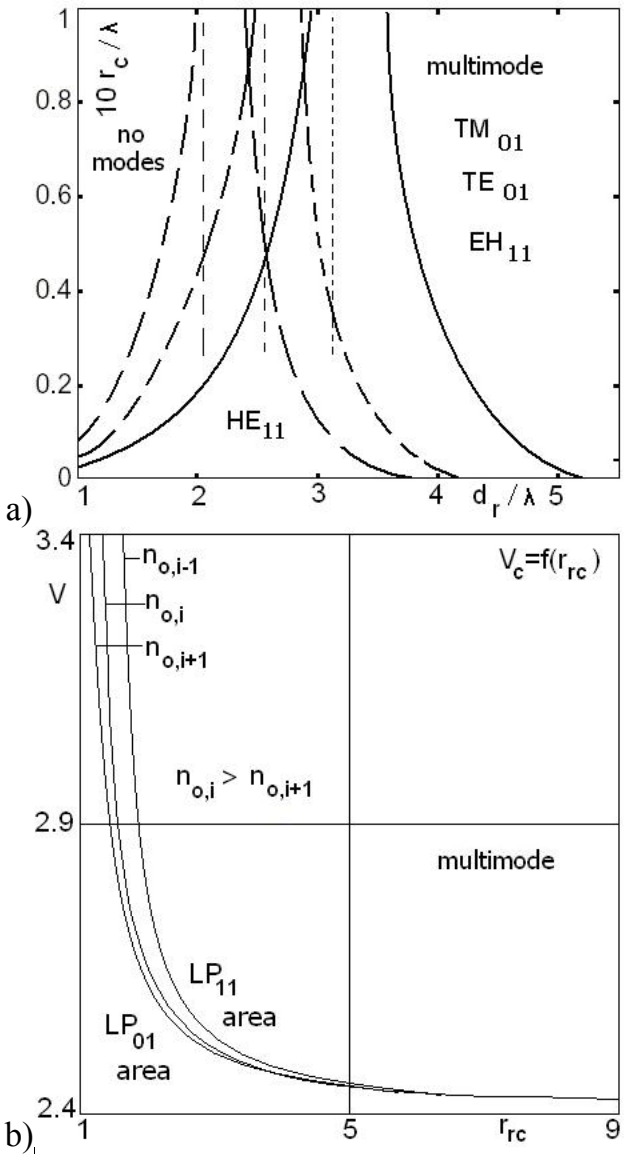

Fig.2. Propagation areas in a COF: no-modes, single-mode, multi-mode as a function of fiber geometry $\mathrm{d}_{\mathrm{T}} / \lambda$ for different NA (a) and $r_{r c}=r_{r} / r_{c}$ and refractive filling of the capillary (b).

Fig. $2 \mathrm{a}$ presents the $\mathrm{HE}_{11}$ mode for increasing values of NA. A COF of high contrast has a lower value of $\beta$ for small values of $r_{c} / \lambda$. The propagation constant is practically nondependent of $r_{c} / \lambda$ for small values of this ratio. Field distribution changes only a little for small capillaries. Fig. $2 b$ presents the propagation constant characteristic for different filling of the capillary. The nondependent variables in the calculated characteristics of a COF are $r_{c} / \lambda, r_{r} / \lambda$, $\beta / \mathrm{k}, \mathrm{n}_{\mathrm{r}}, \mathrm{n}_{\mathrm{p}}$. When the capillary hole is big, the modal structure is similar to a asymmetric planar waveguide (TE and TM modes). A decrease in $r_{c}$, for constant thickness of the ring core leads to the singlemode operation of a COF, Fig.2.

The small diameter of a capillary results in increased separation between the fundamental mode and the next one. $\mathrm{LP}_{01}$ penetrates deeper into the cladding. The cut off frequency of $\mathrm{LP}_{01}$ increases with the dimension of a capillary. The values of derivatives $d n_{\text {eff }} / d d_{r}$ and $d n_{\text {eff }} / d \lambda$, for $n_{\text {eff }} \approx n_{p}$ are small. The potential modal filtering ability of this fiber is weak. The fiber is not a source of modal noise, and is immune to microbending, only for big values of effective refraction (similarly to $\mathrm{W}$ and $\mathrm{M}$ fibers). Effective refraction of fundamental mode decreases for thinner cores and larger $\lambda$, Fig. 3. This dependence is strong for a certain range of capillary diameters, commensurable with $\lambda$. For small and big capillary diameters the sensitivity of the fundamental mode to geometry and refraction of a COF is smaller. Function $\mathrm{n}_{\mathrm{eff}}\left(\mathrm{d}_{\mathrm{r}}\right)_{\mid \mathrm{dc}=\text { const }}$ for a different $\lambda$ is presented in Fig.3a. For a bigger ring core thickness $n_{\text {eff }}$ saturates and next modes appear. For small capillaries, the COF is sensitive to changes in $\lambda$. For big capillaries, around $10 \lambda$, the cut off characteristics of many modes overlap, Fig. $3 \mathrm{~b}$. The separation of modes in the COF of a big hole and high contrast is impossible. The only possible separation is between the following groups: $\mathrm{LP}_{02}, \mathrm{LP}_{03}, \ldots$ and $\mathrm{LP}_{01}$, but not with $\mathrm{LP}_{11}$, $\mathrm{LP}_{21}, \ldots$
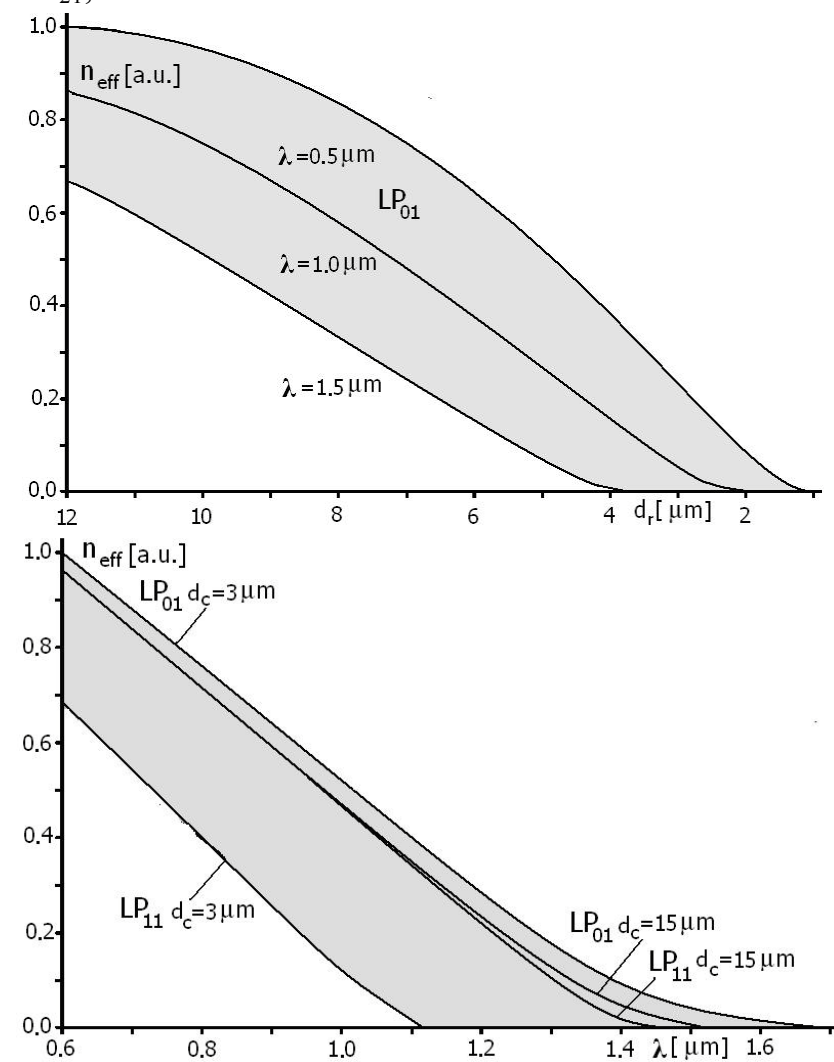

Fig.3. Relative effective refraction of the fundamental mode in a low-refraction COF as a function of ring core thickness for $\mathrm{d}_{\mathrm{c}}=5 \mu \mathrm{m}$ and changing $\lambda(\mathrm{a})$, and as a function of $\lambda$ for $\mathrm{d}_{\mathrm{r}}=5 \mu \mathrm{m}$ and various capillary diameters (b).

Fig.4a presents the singlemode area of a DCOF as a function of parameters $d_{d}$ and $d_{c}$. For example, for $\mathrm{d}_{\mathrm{c}}=5 \mu \mathrm{m}$, a DCOF does not propagate the fundamental mode when the thickness of refractive depression is bigger than $d_{d}>3 \mu \mathrm{m}$. The depression is an effective tool to design a DCOF. Capillary diameter also effectively 
influences the fundamental mode cutoff. Large diameter, up to a certain value, for which COF is singlemode, compensates for large width of the depression. A DCOF possesses two more design parameters which are $n_{d}$ and $\mathrm{d}_{\mathrm{d}}$, in comparison with a COF. For the set core refraction, the cutoff is determined mainly by $\mathrm{d}_{\mathrm{r}}$ and $\mathrm{NA}_{\mathrm{r}}$, and in a lesser degree by $d_{c}, d_{d}$ and $\mathrm{NA}_{d}$. The $\lambda_{\text {cutoff }}$ increases with core thickness and capillary diameter. The values of derivatives $d n_{e f f} d d d_{r}$ and $d n_{e f f} d \lambda$, for $n_{e f f} \approx n_{p}$ are bigger for a DCOF. The cutoff point is well determined. The effective core area is bigger in a DCOF than in a COF for similar geometry and refraction.
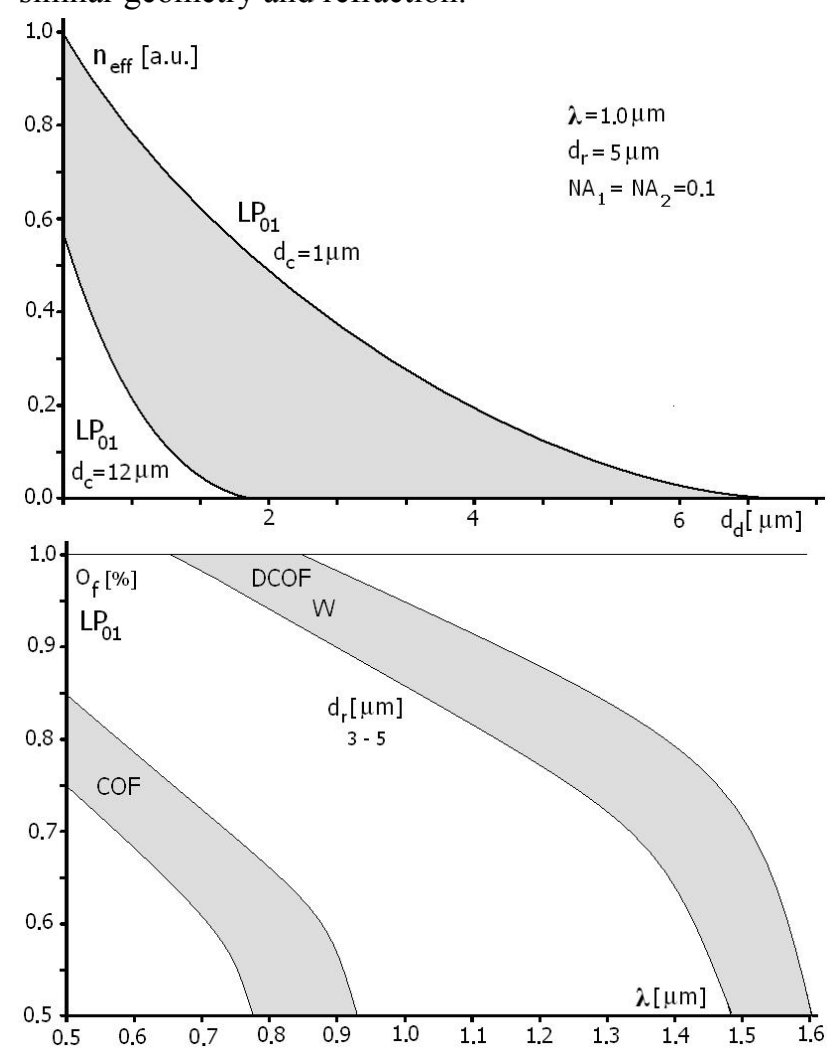

Fig.4. Relative effective refraction of the fundamental mode in a low-refraction DCOF as a function of depression thickness for different capillary diameters (a), Changes in the overlapping of the fundamental mode field with the core area in a COF and a DCOF (and W fiber) as a function of $\lambda$ for different $d_{r}$.

One of the key parameters in active optical fibers, including an active COF, is the overlapping integral of the fundamental modal field with the increased core region designed for high power optical pumping. In a DCOF it is the whole core area including the capillary. The overlapping coefficient in a COF decreases considerably close to cutoff because of the fast spatial dispersion of the modal field. A different situation is in a DCOF, where the modal field is confined additionally by the refractive depression in the cladding, Fig.4b. Additionally, the modal field is less attenuated. The efficiency of a DCOF based laser is higher.
Fig. 5 presents a considerable differences between COF, DCOF and $\mathrm{W}$ fibers in the effective core area. The smallest value is possessed by $\mathrm{W}$ fiber for equivalent geometry and refraction. Simultaneously, W fiber has the widest singlemode area. DCOF and $\mathrm{W}$ fibers are comparable in the immunity to microbending, while COF is less resistant.

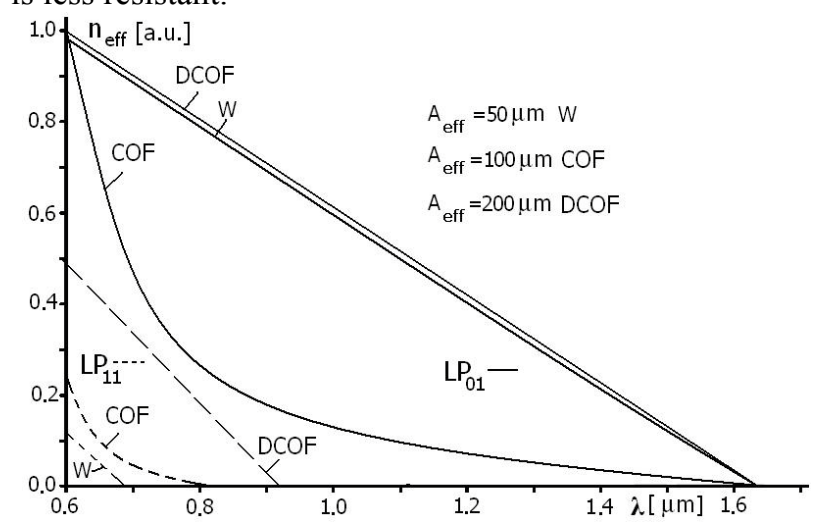

Fig.5. Comparison of effective modal refraction $\mathrm{n}_{\text {eff }}(\lambda)$ for $\mathrm{LP}_{01}$ and $\mathrm{LP}_{11}$ modes for analogous (geometry and refraction) optical fibers: $\mathrm{W}, \mathrm{COF}$ and DCOF.

One of the aspects of modal structure design in a COF is associated with geometry minimization. For certain dimensions (ring core thickness) the fiber does not practically support fundamental mode. Below a certain critical dimension of refractive depression, the cutoff in a DCOF is zero. COFs are also considered for the sub wavelength overall geometry in the range of $r_{c}=(10$ $100) \mathrm{nm}, \mathrm{r}_{\mathrm{r}}=(50-500) \mathrm{nm}$, and $\mathrm{r}_{\mathrm{f}}=(100-500) \mathrm{nm}$. When the capillary is small $(10 \mathrm{~nm})$, the normalized propagation constant B increases with core thickness $(100 \mathrm{~nm})$ and decreases with $\lambda$. When optical cladding thickness is small $(100 \mathrm{~nm})$, the normalized propagation constant B decreases with an increase in capillary radius and $\lambda$. A sub-dimensional COF of larger core thickness has larger B. A considerable difference, between a standard and sub-dimensional COF, in the modal field distribution is on the fiber axis. Small capillaries, below the dimensions of the evanescent field, build up a local field maximum of large gradient on the fiber axis. The shape of a modified dark hollow beam is extremely sensitive to fiber parameters and external reactions. A sub-dimensional COF has large and strongly changeable dispersion. The zero point shifts easily with capillary dimension and core thickness.

\section{References}

[1] V.Neves, et.al., Microwave and Optics Technology Letters 22, 355-357 (1998)

[2] Ch.Y.H.Tsao, et.al., J.Opt.Soc.Am.A. 6, 555-563 (1989).

[3] J.Yin, et.al., Journ. Applied Physics 85, 2473-2481 (1999).

[4] P.R.Chaudhuri, et.al., Optics Communications 228, 285-293 (2003).

[5] A.B.Sotsky, et.al., Opt.Commun. 230, 67-79 (2003).

[6] R.Romaniuk, Bulletin of the Polish Academy of Sciences 56, 1-17 (2008) 